\title{
The relativistic regularities of the periodic radiation of neutron stars
}

\section{Avramenko A.E.}

P.N. Lebedev Physical Institute of the Russian Academy of Sciences, Moscow, Russia;

E-mail: Avramenko<avr@prao.ru>;

The existing numerical models of pulsar timing do not reveal the true accuracy and stability of the periodic radiation of neutron stars. The offered analytical models detect the consistency of the rotation parameters of the pulsar, which confirm the identity of the relativistic pulsar time scales in the coordinate systems and their physical compliance with the barycentric dynamic time and the unreduced topocentric time. A set of parameterized pulsar time scales in spatial systems, oriented in the angular directions to the International Celestial Reference Frame (ICRF), constitute a single astronomical 4-dimensional reference time-space system based on the periodic radiation of the pulsars and the spatial coordinates of the extragalactic sources - quasars.

Keywords: timing of pulsars, rotation parameters, coordinate systems, ephemeris and pulsar time scales.

DOI: $10.18698 / 2309-7604-2015-1-36-48$

\section{Introduction}

Highly magnetized neutron stars, which were discovered as pulsars in 1967, have been proven for the study of a wide variety problems in physics and astrophysics, such as pulsar genesis and neutron star structure, magnetic decay and pulsar braking, properties of the interstellar and the circumstellar medium, long-term stability of its own periodic radiation, etc. Many of results are obtaining by the pulsar timing based on the precise measuring the times-of-arrival (TOAs) of the pulsar signals at the radio telescopes that are located on a rotated Earth orbiting the Sun. Since the observing frame is not inertial, it is provided to transfer the topocentric TOAs measured at the observatory to the center of mass of the solar system (SSB) as the best approximation to an inertial frame available. This transformation includes clock corrections to transfer the measured time to the time standard defined as UTC. Further corrections modify the TOAs at the telescope take into account not uniformly rotating of the Earth and frequency-dependent delay of the pulses caused by the dispersion in the interstellar medium.

The transfer function of TOAs contains a number of astrometric and spin parameters, which are not known a priory and need to be determined precisely in a least squares fit analysis of the measured TOAs. The astrometric parameters include the position of the pulsar, and its proper motion and parallax. Spin parameters are the rotation frequency (or period) of the pulsar and its derivatives. When a timing model including these parameters is fitted successfully, post-fit residuals show a random distribution around zero. Incorrect or incomplete timing models cause systematic components in the post-fit residuals identifying the parameter that needs to be included 
or adjusted. In a separate group include relativistic corrections for a) propagation in the solar system due to the light-travel time from the telescope to SSB; b) an extra delay due to the curvature of space-time in the solar system; c) the combined effect of gravitational redshift and time dilation due to motion of the Earth and other bodies around the Sun [1].

Although the long-term stability of the periodic radiation of pulsars determine their rotation parameters, however they, as a priori unknown values, are fitted along with other parameters. Random variations of the post-fit residuals achieve the millisecond range, which are several orders greater than the variations of modern standards of time.

Our approach, in general, is to find analytical relation of the pulsar time intervals and the physical parameters so that the numerical values of these parameters should be determined and best matched with measured values of the observed intervals. Analytical relations and numerical values should be extended to both, the barycentric and topocentric reference systems. From fitting can be excluded any parameters that can't be obtained directly from the observations.

\section{The parametric consistency of the periodic radiation of pulsars}

It is evident that these problems require a precise analytical solution based on the parameterization of the observed TOAs to avoid the significant effects of unmodeled timing noise caused by random deviations of residuals. Analytical form of the Pulsar Time intervals $P T$ of the periodic radiation, expressed by the rotation parameters of the pulsar, is reduced to Maclaurin power series:

$$
P T\left(P_{0}, \dot{P}, \ddot{P}\right)=P_{0} N+\frac{1}{2} P_{0} \dot{P} N^{2}+\frac{1}{6}\left(P_{0}^{2} \ddot{P}-2 P_{0} \dot{P}^{2}\right) N^{3}, N=1,2,3, \ldots
$$

Here are: $P_{0}, \dot{P}, \ddot{P}-$ the rotation period of the pulsar and its derivatives, $N$ - pulse number in a sequence of radiation events.

Figure 1 shows the graphs of the components of the $P T$ intervals in the power series (1) within of about two years of observations for the specified typical values of the parameters of rotation. This implies that the required values of the rotation period of the pulsar and its derivatives are fixed values, which spread by under certain specified conditions on the duration observations within the borders between the start and end observed events at the current epoch. 


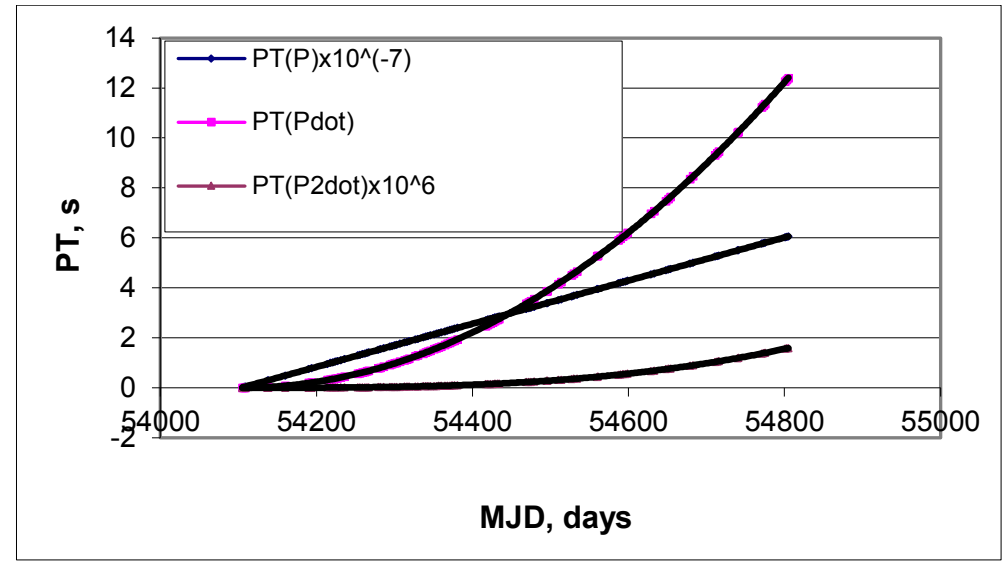

Fig.1. The parametric components of the intervals (1) for typical values of the rotation parameters of the pulsar.

$$
\begin{array}{cl}
\text { Here are: } \quad P T\left(P_{0}\right)=P_{0} N ; \quad & P_{0} \approx 1 \mathrm{~s} \\
P T(\dot{P})=0,5 P_{0} \dot{P} N^{2} ; \quad & \dot{P} \approx 10-15-10-16 \mathrm{~s} \cdot \mathrm{s}^{-1} \\
P T(\ddot{P})=\frac{1}{6}\left(P_{0}^{2} \ddot{P}-2 P_{0} \dot{P}^{2}\right) N^{3} ; \quad \ddot{P} \approx 10-28-10-29 \mathrm{~s}-1 .
\end{array}
$$

Note that the components of the polynomial power series decrease by 5-6 orders of magnitude with increasing the order of the derivatives. By further extrapolating of the rotation parameters the estimated contribution of the third derivative is so small that components of the $P T$ intervals (1), which is defined this derivative within two-year span, does not exceed one nanosecond or less, so that it could not detect by using of the modern physical measurement tools on a background of the observed instrumental noise. Therefore derivatives of the rotation period higher than second order in the model (1) are not considered. That is why the convergence of series is achieved with only three rotation parameters $P_{0}, \dot{P}, \ddot{P}$.

We assumed in the ratio (1), that the rotation parameters of the pulsar, which defines the intervals of pulsar time, are known a priori. However, in reality, these parameters, which are taken for calculations of the intervals, are known only approximately, from the previous observations. Then the problem arises: to retrieve such values of the rotation parameters on the basis of the observed intervals in such a way that they would be consistent with the observed intervals within a selected range of observations.

To resolve this problem, it is required by the measurements of the TOAs to find such values of the observed rotation period and its derivatives, for which the divergence between measured 
TOAs and calculated ones by these parameters, would be the minimum possible within selected range of observations.

If the power series expansion of the form (1) to apply to the measured intervals of pulsar time and associate them with the observed rotation parameters, we obtain the equations whose solutions are observed rotation parameters on the chosen initial epoch observations [2]. This solution determines the numerical values of the parameters of rotation, in which the left and right parts of Eq. (2) coincide within any interval with the estimated accuracy, whatever the initial epoch of observations.

The equation of the observed intervals of $P T$ in accordance with (1) is:

$$
P T_{i}=\left(1+\alpha_{i}\right)\left(P_{0}^{*} N+\frac{1}{2} P_{0}^{*} \dot{P} N^{2}+\frac{1}{6}\left(P_{0}^{* 2} \ddot{P}-2 P_{0}^{*} \dot{P}^{2}\right) N^{3}\right)_{i}
$$

Here are: $P T i$ are the numerical values of the observed intervals obtained from the planetary ephemeris; $P_{0}^{*}, \dot{P}, \ddot{P}$ are the pulsar rotation parameters obtained by solving of the equation (2); $\alpha_{i}$ is divergence of series (2) of the PTi approximated by the rotation parameters of pulsar.

Figure 2 shows the differences between the observed intervals $P T_{o b s}$ and the intervals $P T_{\text {calc }}$ calculated from the observed rotation parameters of the PSR J1509 + 5531:

$$
P T_{o b s}=P T_{i} ; \quad P T_{\text {calc }}=\left(P_{0}^{*} N+\frac{1}{2} P_{0}^{*} \dot{P} N^{2}+\frac{1}{6}\left(P_{0}^{* 2} \ddot{P}-2 P_{0}^{*} \dot{P}^{2}\right) N^{3}\right)_{i}
$$

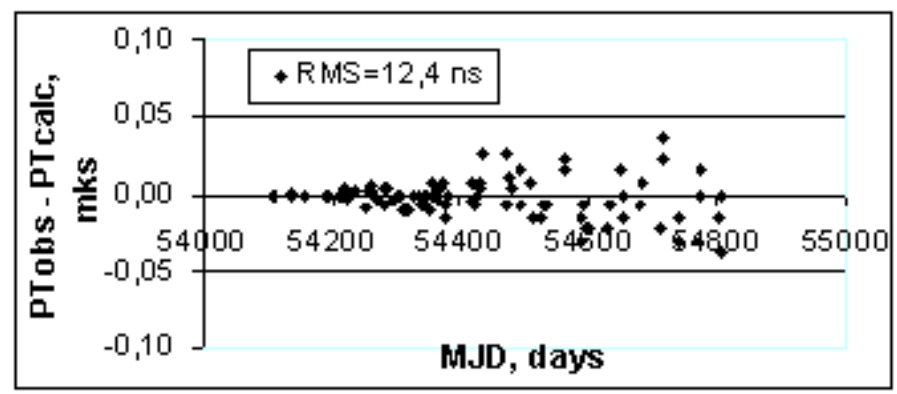

Fig. 2. Convergency of the $P T_{o b s}$ and $P T_{\text {calc }}$ intervals of the PSR J1509+5531.

Graph in the Figure 2 corresponds to the numerical values $P_{0}^{*}=0,739681922904 \mathrm{~s}$ (MJD 49904.0) [3], $\dot{P}=4,99821 \cdot 10^{-15} \mathrm{~s} \cdot \mathrm{s}^{-1}[4], \ddot{P}=3,0669 \cdot 10^{-29} \mathrm{~s}^{-1}$. This value of $\ddot{P}$ obtained by solving the 
equation (2), together with $P_{0}^{*}, \dot{P}$, satisfies the convergence of Eq. (2). The divergence of the $P T$ intervals is determined by the coefficient of variation $\alpha_{i}$. For zero variations, the observed intervals coincide with the intervals, which are determined by the fixed parameters at the initial epoch of observation. In the general case, if $\alpha_{i} \neq 0$, these intervals coincide with the estimated accuracy within any range. As follows from figure 2, divergency of the $P T_{o b s}$ and $P T_{\text {calc }}$ is near $12,4 \mathrm{~ns}$ (rms) within observation range about 2 yrs.

It is obvious, for any choice of the epoch of initial event, the value of period will be different, taking into account the gap between epochs and the derivatives $\dot{P}, \ddot{P}$. The corresponding settings of rotation parameters also satisfy the convergence of the series expansion (3) for any extension in the vicinity specified by the variable $t=P_{0}^{*} N$ :

$$
P(t)=P_{0}^{*}+\dot{P}_{0} \cdot t+\ddot{P} \cdot t^{2} ; t=P_{0}^{*} N, 1<N<\infty .
$$

Here are: $P(t)=P_{0}^{*}+\dot{P} \cdot t ; \quad \dot{P}=\dot{P}_{0}+\ddot{P} \cdot t$.

Values of $N_{i}$, determined by the equation (2), unlike the calculated ratio (1), are not integer due to random variations in the pulse time of arrival (propagation, error of AT, ephemeris of the Solar system, fitting, etc.). Founded in accordance with the equation (2) the real values $N_{i}$ are different from integer value by $\Delta N_{i}=\frac{\Delta \phi(t)_{i}}{2 \pi}$ determined by the observed pulse phase shift $\Delta \phi(t)_{i}=\frac{2 \pi}{P} \Delta t_{i}$ within the current period of rotation.

Real value $\left(N_{i}+\Delta N_{i}\right)$ includes himself in the solution of equation (2), in addition to the $P_{0}^{*}, \dot{P}, \ddot{P}$. It corresponds to the minimum of random variations of the divergence $\alpha_{i}$ and matches the phase of the observed event radiation determined by the stable rotation parameters of the pulsar at any real values of $N_{i}$.

Unmodeled variations of the observed intervals of the coherent pulsar radiation are limited within nanosecond values range, although the scattering of the time of pulse arrival can be up to several milliseconds.

Thus, the pulsar time is synthesized in the parametric form based on the observed rotation period and its derivatives. The numerical values of the parameters of rotation are determined exclusively by the current observational data of timing, any other data (such as residuals) or their 
evaluation (such as RMS of residuals) in addition does not require. The accuracy and stability of the pulsar time is determined by the observed parameters of the pulsar, which are obtained by parametric synthesis, based on the rotational model of pulsar radiation, and are determined from the observed coefficients of the linear approximation intervals within the duration of the observations. Random errors of observed intervals do not exceed (10-18 - 10-19) within the 40year timing continuance of pulsars, which is several orders of magnitude less then this feature of the quantum standards of time.

\section{The space invariance of the observed periodic radiation of pulsars}

Physically pulsar timing measurements are carried out at the Pushchino Radio Observatory at the BSA LPI radio telescope, which operates at frequency range close to $111.3 \mathrm{MHz}$. Pulsar observations were performed on the 512-channel radiometer with a channel bandwidth of $5 \mathrm{kHz}$. The data were sampled at intervals from 0.2048 up to $2.56 \mathrm{~ms}$. The BSA radiotelescope, as a linearly polarized transit antenna with a beam size of about $(3.5 / \cos \delta)$ arcmin, implies the exposure of the observing session ranging from 3 to 11 minutes at different pulsar declinations $\delta$. The topocentric TOAs for each observing session are calculated by cross-correlating the mean pulse profile with a standard low-noise template taking into account of signal delay caused by the dispersion in each channel.

The topocentric TOAs are calculated as a fraction of the current day from their beginning on the date of observation. Numerical values are expressed as a decimal until the 13th sign. Measured in the scale of UTC topocentric TOAs are transformed into the barycenter of the solar system. Barycentric TOAs are counted in seconds, from the beginning of the day of the date of observation. Number format consists of 8 decimal digits, which corresponds to the resolution of TOAs within 10 nanoseconds $\left(10^{-8}\right.$ s).

The numerical conformity of topocentric and barycentric TOAs is not obvious in view of the dynamic divergence of values and the different formats of expression, is provided by planetary ephemeris of the solar system, based on the equations of motion of celestial bodies, taking into account the position and speed of movement observer in space and the limited speed of propagation of the radio signal. The intervals of proper time $\Delta \tau_{1}$ and $\Delta \tau_{2}$ measured by different observers can't be "uniquely" and "naturally" compared to each other. The only way to do so in General Relativity is to define a 4-dimensional relativistic reference system having coordinate time $t$, establish a relativistic procedure of coordinate synchronization of clocks with respe $1 \mathrm{ct}$ to $t$, and convert the 
intervals of proper time $\Delta \tau_{1}$ and $\Delta \tau_{2}$ of each observer into corresponding intervals of coordinate time $\Delta t_{1}$ and $\Delta t_{2}$. These two intervals of coordinate time can indeed be compared directly $[5,6]$.

According to the principle of relativity, which has formulated by Poincare (1906), all physical processes occurring in any inertial system under the same conditions, are identical and correspond to the metric of four-dimensional space-time defined by the invariant interval

$$
(d \sigma)^{2}=c^{2}(d T)^{2}-(d X)^{2}-(d Y)^{2}-(d Z)^{2} .
$$

Spatial coordinates and time in the invariant (5) are related by direct and inverse Lorentz transformations that define common local time $T$ for any points in three-dimensional space:

$$
\begin{aligned}
& \text { Direct: } T^{\prime}=\gamma\left(T-\frac{v}{c^{2}} X\right), X^{\prime}=\gamma(X-v T) ; \\
& \text { Inverse: } T=\gamma\left(\tau+\frac{v}{c^{2}} X^{\prime}\right), X=\gamma\left(X^{\prime}+v \tau\right)
\end{aligned}
$$

Here are: $\gamma=\frac{1}{\sqrt{1-v^{2} / c^{2}}} ; \frac{1}{\gamma} T^{\prime}=\tau ; T^{\prime}$ - changed local time of the $T$.

Lorentz transformations overcome effects of different conditions of observation in the coordinate systems due to movement, current position of the observer, signal propagation time, thus leads physical processes to common conditions of observations [7].

By developing and generalizing the principle of relativity of Poincare, A.A. Logunov (1987) extended it without any changes physical entity to non-inertial reference systems as well, by showing that the interval (5) is invariant in respect any coordinate system [8].

It has been shown (Avramenko, 2009) that the equation of the pulsar time (2) is forminvariant under coordinate transformations, in which the numerical values of the observed rotation period are coincide in the barycentric and topocentric coordinate systems at the same epochs of the local time. Left part of the equation (2) consists the observed topocentric $T T_{o b s}$ or barycentric $T B_{\text {obs }}$ intervals. The right part contains the intervals $T T_{\text {calc }}$ or $T B_{\text {calc }}$, which are calculated by the observed rotation parameters obtained by approximation of $T T_{o b s}$ or $T B_{o b s}$. 
The equation of the $P T$ intervals (2) applied to the accelerated topocentric or inertial barycentric coordinate systems, takes the form, respectively [9]:

$$
\begin{aligned}
& T B_{i}=\left(1+\alpha_{i}\right)\left(P_{0}^{*} N_{B}+0,5 P_{0}^{*} \dot{P} N_{B}^{2}\right)_{i} \\
& T T_{i}=\left(1+\alpha_{i}\right)\left(P_{0}^{*} N_{T}+0,5 P_{0}^{*} \dot{P} N_{T}^{2}\right)_{i}
\end{aligned}
$$

Here are: $P T_{T T}=T T_{\text {calc }} ; \quad P T_{T B}=T B_{\text {calc }}$.

Left parts of the equations (7) are interpreted as observed topocentric TTobs and barycentric TBobs intervals, respectively. The right parts are the intervals TTcalc and TBcalc which are calculated according to the observed rotation parameters of pulsar obtained by approximation of the TTobs and TBobs, respectively.

On the example of the pulsar B0809 +74 Figure 3 shows the intervals TTobs and TBobs and their differences on the two-year observations 2006 - 2008 yrs. at the radio telescope LPA FIAN (Pushchino).
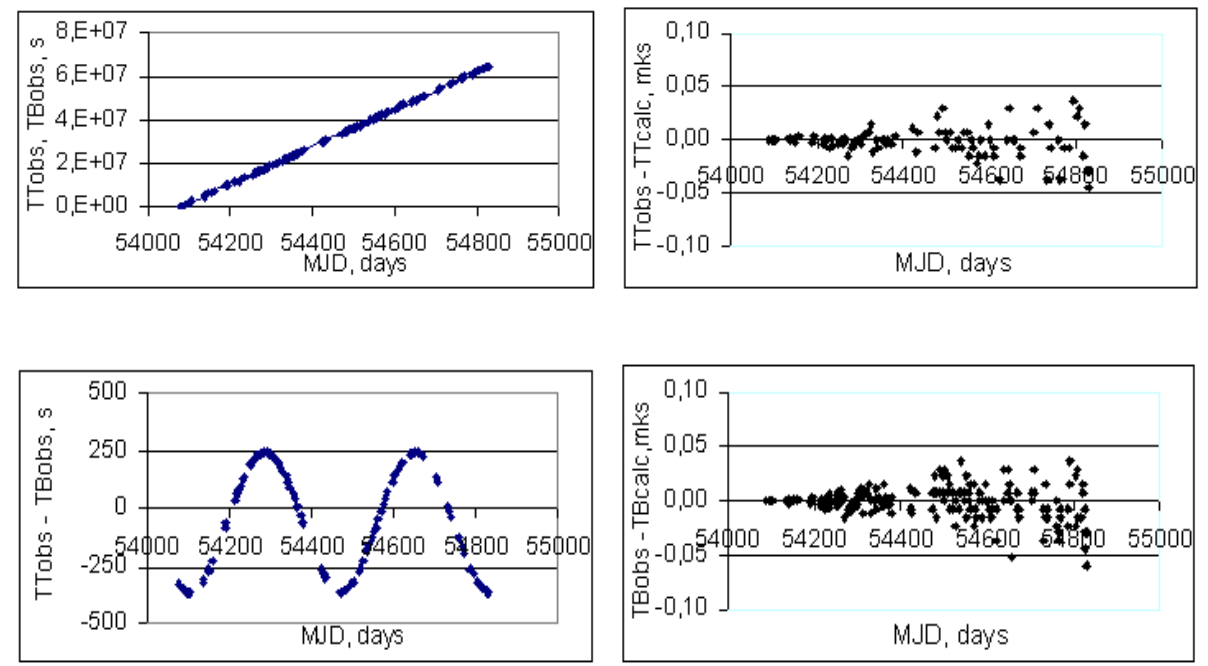

a) the observed intervals (up)

b) differences of the observed and calculated and their difference (down) intervals of $T T$ (up) and $T B($ down)

Fig. 3. Observed topocentric (TT) and baricentric (TB) intervals of the PSR B0809+74 (left), inconsistency of the intervals in the coordinate systems (right).

Monotonically growing intervals TTobs and TBobs have a cyclical changes of their difference (left, up) due to the orbital motion of the Earth around the Sun (left, down). At these intervals in 
accordance with equations (7) have been determined the values of the rotation period $P^{*}$ TT and $P^{*} T B$ on the epoch $M J D$ of the observed pulse counted in local coordinate time scales:

$$
\begin{array}{ll}
P^{*} T T=1.29224151775083 s & \text { at } M J D_{T T}=54080.0098 \\
P^{*} T B=1.29224151775088 s & \text { at } M J D_{T B}=54080.0137
\end{array}
$$

Difference in the values of the observed rotation period in the coordinate systems corresponds to the difference of the epoch of pulse observed in the coordinate systems:

$$
P_{T B}^{*}=P_{T T}^{*}+\dot{P}\left(M J D_{T B}-M J D_{T T}\right) \cdot 86400, \mathrm{~s} .
$$

Here are: $\quad \dot{P}=1,676 \cdot 10^{-16} \mathrm{~s} \cdot \mathrm{s}^{-1}[7] ; \quad$ TTobs - TBobs $=-332.96872 \mathrm{~s}$ (LPA, Fig. 3a)

Note that the value of period in the Cat. [3]: $P=1.292241446861(\ldots) s$ at $M J D=49162.0(\ldots)$ is consistent with the (8), but adopted in the Catalog accuracy is insufficient for nanosecond precision and subnanosecond resolution of the measured intervals of pulsar time.

This is an evidence of the principle of relativity: the physical process of periodic radiation of pulsar observed in barycentric and topocentric coordinate systems under the same conditions, is the same. The numerical values of the observed rotation period are coincide in any coordinate systems at the same epoch of local time.

Figure $3 \mathrm{~b}$ presents the differences TTobs-TTCalc and TBobs-TBcalc that show inconsistency of intervals expressed in the metric of General relativity (GR) based on the numerical ephemeris, and metric of Special relativity (SR) based on the parametric form of $P T$ intervals, in both topocentric and barycentric coordinate systems. The differences of observed and calculated intervals are located in the same range of values in both coordinate systems. Standard statistical evaluation of their small inconsistency is about of $20 \mathrm{~ns}$ within the two-year span. This inconsistency can be associated with the inaccuracy of coordinate transformations of the intervals from metric GR to metric SR and the unmodeled variations of the atomic time scales using for measuring of TOAs.

Consistency of the observed rotation parameters and pulsar time intervals confirms the coherence of the periodic radiation of pulsars. This means equivalence of pulsar time scale in the Cartesian coordinate system, the set of which is a single natural standard 4-dimensional spacetime.

Thus, the intervals of coordinate pulsar time, determined by the observed rotation parameters, are synchronized and can indeed be compared directly in the coordinate systems. 


\section{Metrical unity of time and space in the observed periodic radiation of pulsars}

In order to realize the attainable precision of modern astronomical observations and to understand their physical meaning, it is necessary to use relativistic model of the observed physical processes. Effects of the theory of relativity can not be reduced to only small corrections to the Newtonian models. On the contrary, the whole concept of astronomical reference systems and astronomical observations should be adapted in the framework of the theory of relativity [5].

Modern relativistic systems of astronomical observations are based on the theories of the motion of the solar system bodies in the gravitational field of the Sun and the planets, and on the ephemeris time scales. Definition of ephemeris time scales associated with the geocentric theory of the motion of the Sun and the heliocentric theories of planetary motion. In the dynamic ephemeris model are taken into account the mutual perturbations of large planets, the Moon, the largest asteroids in the framework of General Relativity. Argument the heliocentric ephemeris is barycentric dynamic time TDB. Argument of the geocentric ephemeris of the Sun, the Moon and the planets are terrestrial time TT [10].

In the relativistic model of planetary ephemeris the barycentric TCB and the geocentric TCG coordinate time scales are connected respectively with barycentric dynamic time TDB and terrestrial time TT by a four-dimensional transformations, those at which to implement them could use the International Atomic Time TAI [13]. The basic unit of TAI and TT is the SI second, and the offset between them is conventionally $32.184 \mathrm{~s}$ :

$$
\begin{aligned}
& T C B=T D B+L B(J D-24443144.5) * 86400 \mathrm{c} ; \\
& T C G=T T+L G(J D-24443144.5) * 86400 c ; \\
& T A I-U T C=c(i) ; T T(T A I)=T A I+32,184 c .
\end{aligned}
$$

Here are:

$$
\begin{aligned}
& T C B, T C G \text { - barycentric, geocentric coordinate time, } \\
& T D B \text { - barycentric dynamic time, } \\
& T T \text { - terrestrial time. }
\end{aligned}
$$

Constants of transitions to the coordinate time are equal:

$$
\begin{aligned}
& L G=6,969290134 \cdot 10^{-10} \\
& L B=1,550519768 \cdot 10^{-8}
\end{aligned}
$$


Within the framework of the GRT, the rate of an atomic clock depends on the gravitational potential and its motion with respect to other clocks; thus the timescale entering the equations of motion (and its relationship with TAI) depends on the coordinate system to which the equations refer. In 1991 the IAU adopted resolutions introducing new timescales which all have units of measurement consistent with the unit of time, the SI second. Terrestrial Time (TT) is used for geocentric ephemeris, and Barycentric Dynamical Time $(T D B)$ is used for ephemeris referred to the solar system barycenter. $T D B$ and $T T$ differ by small periodic terms (arising from the transverse Doppler effect and gravitational red-shift experienced by the observer) that depend on the form of the relativistic theory being used: the difference includes an annual sinusoidal term of approximately $1.66 \mathrm{~ms}$ amplitude, planetary terms contributing up to about $20 \mathrm{mks}$, and lunar and diurnal terms contributing up to about 2 mks. $T T$ differs from $T A I$ by a constant offset, which was chosen to give continuity with ephemeris time.

Differences $T T-T D B$ depend on the coordinates of all appropriate bodies the ephemeris, so the more accurate values of these differences are achieved by numerical integration using the following formula for the corresponding ephemeris [11]:

$$
\frac{d(T T-T D B)}{d T D B}=\left(L_{B}+\frac{1}{c^{2}} \alpha^{\prime}\right)\left(1+L_{B}-L_{G}\right)-L_{G}+\frac{1}{c^{4}} \beta^{\prime}
$$

Here $\alpha(t), \beta(t)$ - are mass function, position and speed of massive bodies of the solar system.

Calculated by the formula (10), divergence of the $T T-T D B$ for the ephemeris EPM 2004 and EPM 2008 does not exceed $(1-2)$ ns in the 140-year evaluation interval [12].

The analytical pulsar time scales that are relativistic on physical properties of the observed radiation, are naturally compatible with the local time of the observer in any coordinate reference system. Pulsar time scales TT and TB are agreed with nanosecond accuracy in topocentric (Earth) and the barycentric coordinate observervation systems oriented on the angular axes at the International Celestial Reference frame ICRF-ICRS. The intervals of observed events of pulsar radiation PTobs, calculated by the rotation parameters of pulsar, are the same as the coordinate pulsar time scales $P T_{T T}$ and $P T_{T B}$ in Cartesian topocentric and barycentric coordinate systems of the observer. In contrast to the ephemeris scales $T T$ and $T D B$, the pulsar scales $P T_{T T}$ and $P T_{T B}$ are determined analytically by the observed parameters of pulsar, and their numerical values 
coincide with the observed intervals TTcalc and TBcalc, which are extended to any point in space of topocentric or barycentric Cartesian coordinate system:

$$
T T=P T_{T T}=T T_{\text {calc }} ; T D B=P T_{T B}=T B_{\text {calc }} .
$$

As a result, at any point on the Earth, the location of which is known, the relativistic pulsar time scales synchronize the local atomic scales in the topocentric coordinate system.

So the conversion of the coordinate pulsar time intervals to the form of invariant equations in which the variables are the observable parameters of the pulsar's rotation, revealed the identity of the pulsar time scales in any spatial reference systems that are completely equal for observer. The identity of the metric properties of the pulsar time for both - the numerical and analytical representations of the observed intervals in the spatial reference systems, confirms the equivalence of the metric of general relativity (GRT) and metric of special relativity (SRT). A set of parameterized pulsar time scales in spatial systems, oriented in the angular directions to the International Celestial Reference Frame (ICRF), constitute a single astronomical 4-dimensional reference of time and space based on the periodic radiation of the pulsars and the spatial coordinates of the extragalactic sources - quasars.

\section{Conclusion}

The identity of the pulsar time intervals obtained in numerical form by the planetary ephemeris and approximated in analytical form by the rotation parameters of the pulsar, confirm the equivalence of the metric GR and metric SR.

The rotation parameters of the pulsar obtained from the equations of the observed intervals, are the same in any coordinate system at coincide epoch of local coordinate time, irrespective of choice of the initial epoch and duration of observation.

Intervals of coordinate pulsar time, which are determined by the observed rotation parameters with inconsistency within $10^{-18}-10^{-19}$ for 40 -year duration of observations, are the precise astronomical 4-dimensional relativistic reference measure within the Solar system that are 2-3 orders exceeds of the atomic clock standards.

Thus, coordinate pulsar time scales determined by the observed rotation parameters of the pulsar, are the physical implementation of the barycentric dynamical time $T D B$ and unreduced topocentric time TT. Together with reference ICRF-ICRS, to which are oriented Cartesian observational systems and planetary ephemeris, the parametric pulsar time scales constitute a 
single astronomical 4-dimensional reference system based on the periodic radiation of the pulsars and the spatial coordinates of the quasars.

\section{References}

1. Lorimer D.R., Kramer M. (2005). Handbook of Pulsar Astronomy. Cambridge University Press.

2. Avramenko A.E. (2010). The Observed Rotation Period as an Identifier of the Pulsar Time Properties. Pulsars: Theory, Categories and Applications. Nova Publishers, NY, 61-72.

3. Taylor J.H., Manchester R.N., Lyne A.G. (1993). Catalog of 558 Pulsars. The Astrophys. J. Suppl. Ser., Vol. 88., 529-568.

4. Manchester R.N., Hobbs G.B., Teoh A., Hobbs M. (2005). The ATNF Pulsar Catalogue. Astron. J., Vol. 129., 1993.

5. Klioner S. A. (1995). Relativistic effects in the spatial orientation of the astronomical reference systems. Proc. of the XXVI Radio Astronomical Conference, St.Petersburg, Russia, 294-295.

6.Klioner S. A., Capitaine N., Folkner W. M., Guinot B., Huang T.-Y., Kopeikin S. M., Pitjeva E.V., Seidelmann P. K., Soffel M. H. (2009). Units of relativistic time scales and associated quantities. Proc. of the IAU Symp. 261, 79-84.

7. Poincare H. (1906). Sur la dynamique de l'electron. Rendiconti del Circolo Matematico di Palermo.Vol. XXI.

8. Logunov A.A. (1990). Lectures in Relativity and Gravitation. Pergamon Press.

9. Avramenko A.E. (2009). Form invariance of coordinate pulsar time metrics. Measurement Techniques, Vol. 53, 5, 525-532.

10. Abalakin V.K. (1979). Basics of ephemeris astronomy. Science, Moscow, 448.

11. Klioner S. A. (2008). Relativistic astrometry and astrometric relativity. Proc. IAU Symp. 248, 356-362.

12. Pitjeva E.V. (2012). Fundamental Ephemerides of Planets and the Moon (EPM) of IAA RAS: Their Dynamical Model, Parameters, Accuracy. Proc. of IAA RAS, St.Petersburg, Russia, 149157.

13. Astronomical Yearbook for 2013. (2012). IAA RAS, Science, St.Petersburg, 617-636.

14. Audoin C., Guinot B. (1998). Les fondements de la mesure du temps. Paris: Masson. 